\title{
LOBBYING, SCAMBIO \\ E DEFINIZIONE DEGLI INTERESSI. RIFLESSIONI SUL CASO AMERICANO
}

\author{
di Gigi Graziano
}

\section{Il contesto: pluralismo e lobbying negli Stati Uniti}

L'uso del concetto di scambio come categoria entro la quale sussumere l'attività di lobbying evidenzia un paradosso familiare agli studiosi della politica americana. $\mathrm{Da}$ un lato, nelle sue caratteristiche dominanti la politica americana è per larga parte null'altro che scambio. Il ben noto aforisma di Tip O'Neill (1987), per molti anni Speaker della Camera dei Rappresentanti (1977-1986), secondo il quale «tutta la politica è locale», sottende una nozione nella quale l'ideologia è praticamente assente $\mathrm{e}$ gli interessi particolaristici del tutto dominanti. D'altro canto, non conosco formula che offenderebbe di più operatori e attori del sistema, che la riterrebbero un grossolano travisamento della natura e significato del gioco. Nella misura in cui lobbying è scambio, sarebbero più portati a definirlo come «scambio di informazioni e idee fra Governo e parti private» (CRS Report 1991, 1), capace di infondere nelle politiche pubbliche expertise e consapevole realismo.

Vi sono altre forme di «scambio politico» perfettamente legittime, da alcune scuole di pensiero ritenute anzi il cuore di una politica moderna. Ne è un esempio il neo-corporativismo, nella forma in cui è stato discusso in Occidente e ora nei paesi dell'Est come possibile soluzione ai problemi delle società postcomuniste. Qui lo scambio è un'aperta, legittima forma di compromesso. È questo il significato di scambio politico in Pizzorno (1978), come pure di «scambio politico generalizzato» in Marin

Questo articolo è basato su una ricerca iniziata nel 1987/88 sul lobbying e alcune associazioni lobbistiche a Washington. Una precedente versione è inclusa in un volume curato da Paul Claeys e André-Paul Frognier presso le Edizioni dell'Università di Bruxelles, di prossima pubblicazione.

RIVISTA ITALIANA DI SCIENZA POLITICA / a. XXIII, n. 3, dicembre 1993 
(1990), termine con il quale questo autore si riferisce a forme di «ordine negoziato» fra gli attori economici chiave (Banca centrale, Ministeri chiave, sindacati e organizzazioni imprenditoriali) volte a regolare le variabili macroeconomiche d'interesse comune a tutte queste parti (cfr. Friedberg 1990, 186).

La scena politica americana e il lobbying come sua componente fondamentale differiscono da tale schema neo-corporativo sotto più profili. In primo luogo, come ho argomentato altrove (Graziano 1993), il pluralismo americano è caratterizzato da una concezione molto lata d'interesse, più ampia e comprensiva di quelle che troviamo nelle varianti europee del pluralismo, di matrice cristiano-sociale come socialista. Almeno da Bentley in poi, gli interessi sono stati visti in quella tradizione di pensiero come una realtà ecumenica, espressione di inclinazioni, preferenze, scopi o «passioni» che vanno ben al di là di quantificabili variabili economiche - concezione che trova riscontro nella gamma estremamente diversificata d'interessi organizzati che popola l'attuale scena politica americana, e che comprende chiese, università, minoranze etniche, governi locali e stati, nonché l'intero spettro dei c.d. gruppi d'interesse pubblico o lobbies di cittadini.

Uno «scambio politico generalizzato» ha scarso significato sistemico in una situazione del genere. Così, per fare un esempio, Handgun Control, interessato com'è alla regolamentazione e prevenzione della violenza da armi da fuoco, persegue uno scopo che si colloca al di fuori di qualsiasi accordo generale. Lo stesso vale per l'intera serie di gruppi non economici che si sono moltiplicati negli ultimi decenni. Né i principali gruppi economici hanno mostrato molto interesse per un tale patto, il New Deal rappresentando la più importante eccezione alla regola. Da questo punto di vista, i termini che sembrano meglio adattarsi alla realtà americana sono quelli di «sottosistema lobbistico», come ad esempio la bigher education community che rappresenta a Washington gli interessi delle università e della ricerca, e di policy community, termine che comprende, in aggiunta ad un dato sottosistema lobbistico, le sue controparti nelle agenzie federali e nel Congresso, in particolare i membri influenti delle Commissioni congressuali rilevanti per $i$ gruppi di cui si tratta.

In secondo luogo, diversamente dai sistemi centrati su patti, la politica dei gruppi è, almeno in linea di principio, del tutto competitiva. Nessun altro patto è riconosciuto al di fuori della 
Costituzione e altre regole del gioco che disciplinano il sistema, come la serie di standard etici, legali e congressuali destinati a chi fa attività di lobbying presso il Congresso (cfr. CRS Report, 1991). L'elemento cooperativo, nella misura in cui si manifesta, opera all'interno della «comunità» lobbistica di cui il gruppo fa parte, punto su cui ritornerò; si esplica altresì in coalizioni generalmente temporanee e ben circoscritte nell'oggetto, centrate su ciò che due o più gruppi condividono in termini di interessi per specifiche politiche pubbliche. La specializzazione dei gruppi e la necessità di concentrarsi su un'agenda ben definita, nascono $\mathrm{da}$ e a loro volta alimentano questa struttura frammentata. Da ciò anche il fatto che qualunque interesse l'attore possa avere per il sistema nel suo complesso, non può essere facilmente desunto dai suoi obiettivi come lobbista.

La terza ragione per la quale il lobbismo non può apparire apertamente come scambio sta nei complessi rapporti fra politica delle pressioni e processo democratico. Diversamente dallo scambio sociale e a fortiori dallo scambio economico, il lobbying ha come suo ultimo tipico target pubblici ufficiali, compresi i rappresentanti eletti del popolo. Tanto dice la definizione giuridica, che definisce lobbista qualsiasi organizzazione o persona «che da sola o attraverso un agente..., direttamente o indirettamente sollecita, raccoglie o riceve denaro o qualsiasi altra cosa di valore da usarsi principalmente per contribuire... (a) all'approvazione o al rigetto di un atto del Congresso degli Stati Uniti, (b) ad influenzare... l'approvazione o il rigetto» di un tale atto (Federal Regulation of Lobbying Act 1946, Sec. 307). Il problema è solo leggermente meno delicato quando la pressione è diretta, anziché sul Congresso, su amministratori e agenzie federali, formalmente esclusi dalla definizione legale ma di fatto centrali al funzionamento dell'intero sistema.

In effetti, per quanto pratica diffusa e vecchia di decenni, la presenza di lobbisti nei corridoi del Congresso non è mai stata riconciliata in modo soddisfacente con $i$ requisiti formali del processo legislativo. Ciò è particolarmente vero quando lo staff di associazioni redige parti o l'intero testo di una legge, nelle varie fasi di un processo che segue passo a passo - dalla presentazione del progetto di legge, che può «sponsorizzare», all'esame in Commissione con relativi emendamenti e voto in aula, fino alla «conferenza» fra delegazioni di Camera e Senato nel caso normale di discrepanza fra i testi dei due rami. So ad esempio che intere sezioni del Higher Education Reauthoriza- 
tion Act (1992) - una legge vitale per il sistema universitario americano e i finanziamenti federali di cui beneficia - sono state materialmente scritte o fortemente influenzate da una dozzina di associazioni rappresentanti università e colleges, attraverso «raccomandazioni» offerte articolo per articolo alla commissione competente a redigerne il testo, il House Postsecondary Subcommittee. Il diniego in tali casi dell'esistenza di qualunque scambio è ancora più comprensibile, giacché ogni accordo nella stesura della legge (non solo nella sua sponsorizzazione da parte di un gruppo) costituirebbe una seria intromissione nell'attività del Congresso come organo democraticamente eletto.

Quanto precede serve al contempo ad introdurre la nozione di lobbying com'è usata in questo saggio e ad evidenziare alcuni dei problemi che incontriamo quando cerchiamo di ricomprenderla nell'idea di scambio. Va subito aggiunto, peraltro, che in quanto comportamento o rapporto interpersonale e interorganizzativo, il lobbying s'ispira in gran parte a un'etica e logica dello scambio non dissimili da quelle delineate in un certo numero di studi, in particolare il lavoro pionieristico di Blau (1964). Da un lato, gratitudine e fiducia personale sono fondamentali in entrambi, come «cemento» di rapporti che non possono essere racchiusi in precisi termini contrattuali. Nell'attività di lobbying, gratitudine e fiducia sono - in aggiunta alla competenza in specifiche policy areas - la chiave dell'accesso, cioè della capacità di essere efficacemente sentiti dai decisori quando prendono decisioni rilevanti per il gruppo.

Come vedremo più in dettaglio nel terzo paragrafo, la teoria di Blau consente altresì di penetrare nel tipo di reciprocità sotteso al lobbying. Non diversamente dallo scambio sociale, il lobbying ha un orientamento strumentale: qui gran parte delle aspettative della clientela (membri interni del gruppo o coloro da cui origina la committenza in caso di lobbisti indipendenti) fa perno sulla capacità di estrarre tempestive e appropriate risposte da pubblici ufficiali e pubbliche istituzioni, attraverso un repertorio di strumenti, strategie e risorse su cui ritornerò (vedi terzo paragrafo).

Infine, la teoria di Blau attira la nostra attenzione sul problema dell'istituzionalizzazione e legittimazione. Il lobbying negli USA è ormai un sistema diffuso a livello locale, statale e federale relativamente ben istituzionalizzato, le cui regole e tecniche sono codificate in manuali (cfr. Zorack 1990 e molte altre guide pratiche) e insegnate in reputate università e centri di ser- 
vizi, il più noto dei quali è quello gestito a Washington da «Congressional Quarterly». Dovremo quindi investigare alcuni dei meccanismi e vincoli che hanno portato alla regolazione dell'attività, facendo del lobbying più che in ogni altro paese una parte riconosciuta anche se non sempre accettata del sistema politico americano.

Prima di trattare del lobbying alla luce di questa teoria vorrei aggiungere qualche osservazione sulla categoria dello scambio applicata alla politica.

Scambio economico, scambio sociale e politica. La definizione degli interessi

Nel suo significato originario, scambio è un concetto economico ${ }^{1}$. Solo recentemente, negli ultimi due o tre decenni, è diventato un paradigma sempre più importante nello studio della politica e della società. Una possibile ragione di ciò sta nel fatto che l'idea di scambio attira l'attenzione sulla crescente interazione all'interno della società e fra politica e società, in una situazione di sempre più accentuata importanza del consenso sociale per il funzionamento del sistema. Il consenso, tuttavia, è assicurato attraverso nuovi canali e fa riferimento anzitutto agli interessi fortemente organizzati, cosa vera tanto per il neo-corporativismo che per il pluralismo. È un consenso selettivo, che integra ma anche contrasta con il principio di eguaglianza e inclusione che è alla base del modello democratico.

Tutto ciò semplicemente per ricordare che quando si passa dagli interessi sociali alla loro rappresentanza politica entro un contesto democratico, ci si trova di fronte a seri dilemmi normativi, ampiamente discussi nella letteratura «revisionista» degli anni sessanta fortemente critica del pluralismo (Wolin, Schattschneider, Olson, Grant McConnell, Lowi). Questi dilemmi non possono essere qui discussi estesamente; ci si deve limitare ad alcuni dei problemi analitici che incontriamo allorché la nozione di scambio è trasferita dall'economia alla società e poi alla politica.

Sono tre i caratteri distintivi dello scambio economico: 1)

1 Il concetto è stato ampiamente usato anche al di fuori dell'economia, ad esempio negli studi antropologici. Ma lo scambio è stato essenzialmente elaborato dagli economisti. 
benefici possono essere ottenuti attraverso l'interazione sociale per via di scambi con altri attori, non in isolamento dalla società; 2) self-interest o interesse personale come forza che motiva le persone a scambiare; 3) lo scambio economico presuppone un'economia monetaria, ossia «un mercato nel quale beni e servizi scambiati sono valutati sulla base di un unico mezzo di scambio» (Baldwin 1990, 105). In altri termini, lo scambio economico presuppone l'esistenza di qualche forma di moneta.

Nella vita sociale, alcuni di questi requisiti ricorrono in modo incompleto, mentre altri, come la presenza di un mezzo generalizzato con cui comprare merci e ripagare debiti, sono del tutto assenti. È l'assenza di un equivalente sociale del denaro, unitamente alla natura generica e non specifica degli obblighi in cui si incorre per effetto dello scambio sociale, che più chiaramente distinguono quest'ultimo dallo scambio economico. $\mathrm{Ma}$ atteniamoci per il momento all'interesse personale come motivazione dominante, in effetti esclusiva in un mondo dominato dall'impulso economico. Come vedremo, nella vita sociale il self-interest è qualcosa di più indeterminato e dinamico rispetto alla nozione che ne ha la teoria economica, e si definisce come realtà operativa (che muove cioè all'azione) attraverso processi nei quali l'organizzazione del gruppo ed il suo staff svolgono un ruolo centrale.

Olson ha persuasivamente mostrato quanto sia utile applicare il concetto economico di self-interest all'analisi delle associazioni. La sua teoria ha profondamente innovato lo studio dei gruppi. Le sue argomentazioni valgono però solo quando 1) vengono riferite all'interesse personale di individui, ossia alle opzioni di attori individuali ${ }^{2}$, e 2) nella misura in cui le motivazioni extra-economiche sono escluse dall'analisi. Si noti che Olson non esclude affatto l'altruismo o motivazioni filantropiche dal novero delle forze che motivano all'azione; semplicemente, in quanto economista intento ad offrire una teoria economica dell'azione collettiva tiene fuori dalla teoria motivazioni e forme di associazione che non possono essere ricondotte al self-interest.

Abbiamo dunque nella società l'importante problema dell'«altruismo», che deve essere una delle forze sociali sottese alla

${ }^{2}$ Olson tratta anche, ed estesamente, di organizzazioni (imprese, sindacati, associazioni) non solo di individui, ma sulla base di convenienze riconducibili alle motivazioni che muovono l'uomo economico. 
forte proliferazione di gruppi d'interesse pubblico negli USA specie a partire dagli anni settanta, e di altri fenomeni come il volontariato in Italia e altrove. La sola pertinente osservazione qui è che fra altruismo e self-interest ci sono molti ponti. Si consideri un'associazione come Handgun Control, una delle più efficaci e temute «lobby di cittadini», il cui piccolo nucleo di attivisti è composto quasi esclusivamente da persone che hanno personalmente sofferto ferite da arma da fuoco o i cui congiunti sono stati così feriti o uccisi. (L'attuale presidente di Handgun Control, Sarah Brady, è moglie dell'ex segretario stampa di Reagan gravemente ferito durante l'attentato a Reagan nel 1981.) Questi attivisti sono motivati da self-interest o da altruismo? Né il caso è unico: l'aborto è un altro esempio di «interesse» in cui motivazioni personali e impegno per una causa pubblica convergono rafforzandosi a vicenda.

Non solo il mix di motivazioni è incerto, circostanza che fa del self-interest un fondamento assai fragile di un comportamento sociale «razionale». Ugualmente se non più importanti sono i complessi processi psicologici attraverso i quali una persona giunge a definire a scopi pratici la propria sfera di interessi, siano essi egoistici o filantropici. La più importante variabile interveniente è sotto questo profilo l'organizzazione. Gli attivisti di Handgun Control si riferiscono a questo aspetto del processo con il termine «incanalamento» (channelling), la capacità dell'organizzazione di trasformare la sofferenza in stimolo attivo all'azione sociale, la vittimizzazione in un interesse intorno a cui organizzare l'azione politica. È questo campo precipuo d'attività dello staff del gruppo che contribuisce da Washington a formulare il problema della vittima in termini che conducano all'azione, suggerendo cosa fare, a chi scrivere, come muoversi in rapporto a quale provvedimento in discussione. Sottolinea il ruolo decisivo della professionalizzazione e dello staff professionale, non solo nella conduzione dell'organizzazione ma nel forgiare la stessa mappa di interessi di cui le organizzazioni lobbistiche si fanno carico.

Le difficoltà crescono quando la nozione di self-interest è riferita ad organizzazioni complesse. Anche qui lo staff definisce le issues e la loro percezione, generalmente tramite «Public Affairs Reports» e altre forme di comunicazione periodica con i membri. In aggiunta, avviene spesso che i membri o le istituzioni membri di un'associazione abbiano interessi difficilmente aggregabili in un corpo coerente, con conflitti fra ade- 
renti o anche al loro interno. Si pensi, per esemplificare, al fascio di interessi diversificati che fanno capo all'istituzione università (di amministratori, personale insegnante, ricercatori, studenti e, sotto il profilo funzionale, quelli attinenti alla ricerca e fondi relativi, rispetto a quelli dell'insegnamento, ecc.), interessi fra cui l'associazione non media ma di cui deve tenere conto nella propria opera di rappresentanza. In casi come questi trovo utile pensare a un gruppo come ad un insieme di «clientele», ciascuna con una propria agenda e priorità, e agli obiettivi e scelte dell'associazione come risultato di accordi «diplomatici» che riflettono più o meno fedelmente l'equilibrio interno delle forze.

Fin qui la dinamica interna al gruppo di un processo che ha però anche importanti risvolti esterni. Nella genesi e definizione degli interessi politici il Governo svolge un ruolo essenziale. Lo staff e l'organizzazione materiale della rappresentanza a Washington, una volta che un'associazione abbia deciso questo passo, sono il motore operativo dell'aspetto politico dell'attività di gruppo, della sua interazione cioè con le attività e istituzioni di Governo. Come ho scritto altrove (Graziano 1993), una lobby non ha realtà ontologica a prescindere dai suoi rapporti con il potere politico -- dimensione questa curiosamente omessa nella teoria di Olson, che si conferma teoria intra-organizzativa, nella quale lo stato si pone come variabile estrinseca, oggetto di pressioni da parte dei gruppi ma sostanzialmente estraneo ai processi che possono chiamarli in vita ${ }^{3}$.

In effetti, chi studia il processo di politicizzazione delle associazioni nel tempo sa che il ruolo del Governo è essenziale. Il Governo non è solo una realtà reattiva alle pressioni dei gruppi: con la propria azione politica - agevolazioni fiscali (che gli americani chiamano significativamente tax expenditures), creazione di entitlements (pensioni, ecc.), politiche regolative di vario tipo, ecc. - crea utilità e disutilità di cui i gruppi possono usufruire (o evitare) solo con un'azione lobbistica. Spesso poi l'azione di gruppo serve non già ad ottenere dal Governo una nuova politica, ma ad evitare $\mathrm{i}$ danni e costi derivanti da una

${ }^{3}$ Olson considera il ruolo dello stato nella nascita di alcuni gruppi. Ma tali gruppi sorgono o per convenienze del Governo alla ricerca di interiocutori organizzati (caso della Camera di Commercio USA), o per processi indotti dalla sua azione istituzionale (caso della Farm Bureau Federation organizzatasi a partire dai county agents, operatori federali mandati nelle campagne per la modernizzazione dell'agricoltura). 
politica già statuita - e l'azione collettiva volta ad evitare un danno certo ed evidente è più facile da «vendere» ai propri associati rispetto a quella volta a strappare nuove politiche pubbliche.

Possiamo generalizzare il problema formulandolo così: il problema del free-riding, e quello connesso dell'attitudine di un interesse a trovare forma organizzata, cambia completamente di segno dopo l'azione del Governo (Salisbury 1990). Dopo che una legge è stata varata o un regolamento emesso, cambiano due cose, che rovesciano lo schema di convenienze delineato da Olson: 1) la «passività razionale» di chi non ha interesse a mobilitarsi per una politica comune, ora contribuisce a sostenerla: pochi saranno infatti spinti ad organizzarsi per far abrogare quei provvedimenti, così come pochi - vigendo la razionalità postulata da Olson - avevano interesse ad organizzarsi perché venissero in essere. L'inerzia, come sempre, gioca a favore degli interessi costituiti; 2) nella realtà del welfare state, molti provvedimenti hanno natura tale da aumentare grandemente la consapevolezza dei beneficiari che qualcosa di vitale è in gioco, qualcosa per cui vale la pena, anzi è decisivo, organizzarsi politicamente attraverso l'azione di gruppo (Salisbury 1990).

La trasformazione storica di molte associazioni americane in lobbies, processo vistosamente accentuatosi a partire dagli anni settanta, risponde perfettamente a questo schema, per il quale gli interessi in politica sono realtà emergenti e dinamiche, non dati precostituiti. Valga come esempio il caso di una delle associazioni studiate da chi scrive: l'Association of American Universities, sorta nel 1900 come club di presidenti di 14 fra le più prestigiose università americane, e trasformatasi negli anni settanta in lobby, sulla scia di un processo di politicizzazione che ha coinvolto gran parte della bigher education community e le sue varie associazioni. Nel rapporto che segnò questo passaggio di AAU da club a lobby, con staff accresciuto e presidente fulltime a Washington, si legge:

... varie branche e agenzie del Governo federale stanno considerando una lunga lista di materie di enorme significato per le università impegnate nella ricerca - sovvenzioni alla undergraduate education, riforma fiscale,... legislazione su brevetti e copyright, peer review, affirmative action, ricerca biomedica, quote sui fondi di ricerca attribuibili alle università a titolo di spese generali... Quasi sistematicamente si levano forti voci a favore di azioni federali che potrebbero seriamente compromettere gli interessi delle università. Per queste ragioni, dobbiamo assicurarci che i nostri interessi siano chiaramente espressi 
e efficacemente presentati ai funzionari... responsabili delle decisioni e politiche pubbliche». (Rapporto Bok 1976, 2) ${ }^{4}$

Vale concludere sul punto. Il processo di definizione degli interessi, e le decisioni relative alla convenienza di dar vita a gruppi capaci di tutelarli politicamente, sembrano essere frutto di un'interazione complessa fra individui, gruppo e Governo. C'è un costo di partecipazione, su cui Olson giustamente insiste, ma c'è anche un costo da inazione. Così nel citato Rapporto Bok sulla ristrutturazione di AAU, accanto ai costi dell'operazione e ai rischi di uno staff che avrebbe inevitabilmente vieppiù marcato la propria autonomia dai membri, si faceva rilevare come costi e rischi «di un'efficace rappresentanza [fossero] insignificanti rispetto alla dimensione e ampiezza dell'intervento federale» (Rapporto Bok 1976, 12). L'altra alternativa, di lasciare che ciascuna università facesse individualmente attività di lobbying, era non solo costosa ma inefficace.

Vale aggiungere che lo studio storico delle associazioni e del loro processo di politicizzazione - processo che non risponde solo a dati strutturali come vorrebbe Olson (gruppi di pochi membri e sistemi di pochi gruppi), ma anche congiunturali-processuali come abbiamo visto - evidenzia l'utilità di disaggregare la nozione d'interesse. Trovo utile distinguere tre livelli: 1) valori o ispirazione programmatica di fondo di un'associazione; nel caso di AAU, lo scopo per cui si pensò di riunire le università che allora impartivano corsi di $\mathrm{PhD}$. era di promuovere la graduate education in America, in un tempo in cui gran parte degli studenti USA veniva ad addottorarsi in Europa e specialmente in Germania, a Berlino; 2) i settori o policy issues su cui il gruppo sceglie di concentrare la propria attività, e che ne segnano la specializzazione all'interno della comunità d'appartenenza (nel caso di AAU si tratta di graduate education, biblioteche di ricerca, ricerca finanziata con fondi federali); 3) obiettivi contingenti, che lo staff individua in rapporto all'evolversi della congiuntura politica (iniziative del Governo, del Congresso, ecc.) e altre considerazioni di strategia e tattica. Questi diversi livelli permettono di meglio cogliere le trasformazioni dei gruppi in rapporto al mutare dei loro scopi nel tempo.

${ }^{4}$ Il rapporto prende nome dall'allora Presidente di Harvard, Derek Bok, coordinatore del gruppo di lavoro incaricato di redigerlo. 


\section{Il lobbying come scambio}

Nelle precedenti sezioni ho argomentato che sebbene il lobbying non possa farsi facilmente rientrare nella categoria dello scambio politico esso condivide con lo scambio sociale molti tratti comportamentali. Così, ad esempio, molti lobbisti danno vita a propri PAC (Political Action Committees) e contribuiscono alle campagne elettorali al fine di ottenere più facile «accesso» ai Congressmen beneficiati (Sorauf 1993). Inoltre, anche quando non sollecitati, i gruppi spesso forniscono regolarmente a legislatori e amministratori informazioni tecniche aggiornate nei campi di rispettiva competenza, nell'intento di tenere i canali aperti e rendere un servizio utile che sarà sperabilmente contraccambiato. Va aggiunto che Congressmen e massimi responsabili delle agenzie federali sono soltanto uno e non fra i più frequenti referenti dei gruppi. L'interazione avviene generalmente con lo staff dei rispettivi uffici, ed è particolarmente a questo livello che il lobbying tende ad assumere la forma di scambio.

Nello studio della morfologia del fenomeno, peraltro, la prima domanda che vien fatto di porsi è quella di sapere perché un diritto costituzionalmente protetto si sia largamente sviluppato come scambio informale. Oggi si tende generalmente a inquadrare il lobbying nel diritto di petizione enunciato nel primo emendamento alla Costituzione americana (right «to petition the Government for a redress of grievances») - in netto contrasto, va notato, con la tradizione europea, specialmente quella rivoluzionaria-francese, che esplicitamente vietava ogni forma di petizione all'Amministrazione sotto pena di nullità dell'atto (cosiddetta Legge Le Chapelier, 1791). Né il gioco è completamente informale, giacché vi sono procedure e arene ufficiali attraverso le quali gli interessi organizzati possono farsi politicamente valere, come ad esempio le audizioni congressuali 5 .

La risposta sta nella natura intrinsecamente informale della rappresentanza degli interessi in un contesto pluralista. Da un lato, anche quando il gruppo è formalmente organizzato, non è sempre facile misurare il suo reale grado di rappresentatività.

5 Zorack (1990) tratta dei gruppi in rapporto alle fonti di produzione legislativa, inquadrando l'attività di sponsorizzazione di progetti di legge da parte di associazioni nel diritto di petizione. 
Quando ad esempio Handgun Control vanta di avere «un milione di membri», l'esatto significato della formula non è chiaro, data l'assenza di qualsiasi definizione formale dello statuto di «membro». (In effetti, nel 1992 c'erano circa 300.000 contribuenti al gruppo che versavano periodicamente contributi in danaro ogni 3-4 anni.) Lo stesso può dirsi di gran parte delle associazioni con membri. Inoltre, la stessa nozione di rappresentante è complessa: in aggiunta ai suoi rappresentanti ufficiali (presidente dell'associazione, responsabili per i rapporti federali o legislativi, ecc.), le associazioni possono e spesso fanno ricorso a loro membri autorevoli come portavoce. Per alcune materie, il miglior lobbista dell'AAU è il presidente di Harvard, con tutta l'autorità e il peso di quell'istituzione, pur tenuto conto del grande prestigio e autorevolezza della persona che ha ricoperto fino ai primi del '93 l'ufficio di presidente dell'associazione. Infine, quali gruppi fra quelli esistenti debbano essere consultati dal Governo o dal Congresso, a quale stadio del processo e con quali prerogative sono altrettante questioni che non trovano risposta negli ordinamenti formali.

Gli attori implicati nello scambio possono quindi variare. Il gruppo opera anzitutto attraverso i suoi rappresentanti ufficiali, la maggior parte dei quali è installata a Washington nella sede delle rispettive organizzazioni: attualmente circa 14.000 persone, siano essi registrati come lobbisti o meno (cfr. «Washington Representatives» 1992). Spesso si fa ricorso a lobbisti esterni (o indipendenti) ingaggiati per specifiche campagne o su base continuativa. $\mathrm{Ma}$, come ho detto, le organizzazioni d'interessi tendono a scegliere qualsiasi membro, persona o esperto in grado di massimizzare la loro influenza in rapporto alla funzione da compiere. Nelle audizioni indette dalle Commissioni del Congresso, ad esempio, le associazioni hanno tendenza a scegliere come testimoni personaggi influenti nel collegio elettorale del presidente della Commissione o di altri membri chiave (presidente dell'Università dello Stato del Chairman, responsabili di grosse imprese locali, ecc.). Nei rapporti con le Agenzie federali, di natura più tecnica, i contatti sono generalmente tenuti da staff interni al gruppo, specializzati nell'area di attività dell'Agenzia. Altre volte ancora, la rappresentanza è delegata ad altre associazioni nel quadro della «politica delle coalizioni», su cui ritornerò. Spesso si ricorre ad esperti quando si vogliono enfatizzare gli aspetti tecnico-professionali delle materie in esame, le ricadute sulla comunità scientifica, ecc. Talvolta infine si 
ricorre a semplici utenti che hanno un'esperienza personale da raccontare connessa al provvedimento in esame, che massimizza le chances di trasformare la testimonianza in forte appello emotivo. La varietà di situazioni è pressoché infinita, a conferma del fatto che è la ricerca dell'influenza più che la rappresentanza formale che guida le opzioni di un gruppo.

Mentre tutti questi attori sono importanti, i veri protagonisti dal lato delle associazioni sono i membri del loro staff burocratico. Nulla può eguagliare la competenza tecnica e l'intelligenza politica di un esperto fiscale di un'associazione in fatto di politica fiscale nei suoi rapporti con gli interessi associativi. Competenza professionale e contatti quotidiani con il Congresso e l'Amministrazione sono le risorse fondamentali di questo tipo di personale. Nessun Congressman o membro del suo staff a Washington (sino a 18 collaboratori per ciascun deputato, un numero più elevato per i senatori a seconda della dimensione dello stato di provenienza) è probabile maturi lo stesso tipo di perizia. Né è semplicemente questione di superiorità tecnica: chi dedica tutto il proprio tempo al monitoraggio ad esempio della politica fiscale dentro e fuori dal Congresso sarà in grado $\mathrm{di}$ acquisire maggiori conoscenze in fatto di schieramenti correnti, possibili co-sponsors di nuove leggi - in una parola circa la politica del problema - della maggior parte dei Congressmen. Di qui una dipendenza dovuta alla specializzazione del lobbista, tanto maggiore quanto più forte è il grado di fiducia personale che lega l'uomo politico al «suo» esperto.

Lo staff dei gruppi e le associazioni operano spesso attraverso coalizioni, strategia fondamentale nella politica delle pressioni. Le issues in rapporto alle quali i gruppi si coalizzano possono essere complesse e numerose o consistere ad esempio in un singolo articolo delle leggi fiscali. Le coalizioni possono contare decine, talvolta centinaia di membri, in genere altre associazioni, o essere di dimensioni più ridotte; informali o più o meno istituzionalizzate con un proprio ufficio di coordinamento e risorse autonome (la norma è che un'associazione assume la leadership e coordina le altre). Ancora: essere attive per l'intero arco dell'anno o solo in particolari momenti del calendario del Congresso, tipicamente durante il processo di formazione del bilancio. Qualunque ne sia forma e consistenza, la funzione principale della coalizione non è semplicemente quella di mostrare forza numerica (molti gruppi); ugualmente se non più importante è l'autorevolezza e diversità dei membri, prova che 
l'agenda politica per cui ci si batte sta a cuore ad un'ampia e diversificata fetta dell'opinione pubblica, forse più ampia degli interessi direttamente rappresentati nella coalizione. Infine, la presenza di altri partner dà più peso e credibilità alle richieste di un gruppo, che vede la propria causa perorata e legittimata da altri (industrie bigh-tech alleate all'università che argomentano a favore di maggiori fondi per la ricerca accademica; associazioni di poliziotti arruolate a sostegno dei provvedimenti per cui si batte Handgun Control, ecc.).

Per concludere sul ruolo politico del personale delle associazioni va aggiunto che lo staff spesso opera come una specie di think-tank dell'ufficio di un Congressman o di una Commissione parlamentare, redigendo leggi, suggerendo emendamenti e svolgendo altre funzioni semi-pubbliche. Almeno in un'occasione a mia conoscenza, il già citato Higher Education Reautborization Act (1992), lo staff della Commissione incaricata di stenderne il testo si è rivolto ad una delle associazioni affinché mediasse i conflitti sorti all'interno di un importante settore della comunità universitaria, investendo quindi l'associazione di funzioni tecnico-politiche che hanno trovato poi riflesso nel contenuto della legislazione. Infine, al gruppo può essere rivolta la richiesta o l'associazione può spontaneamente offrirsi di organizzare sostegno per un legislatore amico in cerca di co-sponsors per un proprio progetto di legge o mobilitare la policy community e le sue associazioni a sostegno di tali iniziative, con incontri con il Congressman al Congresso o in altra sede, e in altri modi ancora.

Nel gioco lobbistico entrano due altre risorse importanti: il c.d. grassroots lobbying o lobbying indiretto e i mass media. La mobilitazione a sostegno delle finalità del gruppo prende generalmente la forma di una strategia a due livelli: pressione al centro attraverso lo staff di Washington e pressione alla base, a livello del collegio elettorale del membro del Congresso che si vuole influenzare. Il peso relativo delle due strategie varia a seconda della natura del gruppo. Gruppi d'interesse pubblico come Common Cause o NAACP (National Association for the Advancement of Colored People) o lobbies di ecologisti, ricchi in risorse umane ma in genere finanziariamente deboli, hanno tendenza a dare a queste tecniche un peso maggiore, imperniando su di esse le proprie strategie di mobilitazione. Diramano tempestive «allerte», tengono i loro membri informati su ciò che è in discussione al Congresso e le varie issues all'ordine del 
giorno a Washington, consigliano sull'opportunità d'intervenire in incontri locali una volta che il Congressman è nel proprio collegio, ecc. Tutto questo, va notato, avviene in forme e tempi che sarebbero tecnicamente impossibili, oltre che dispendiosissimi, senza le nuove tecnologie della comunicazione (computer e reti interattive, posta elettronica, linee telefoniche a tariffa ridotta, ecc.).

Nozione vaga che sfuma alquanto i confini fra lobbying e comunicazione, il grassroots lobbying ha oggi una definizione più precisa grazie alla normativa fiscale. Le Internal Revenue Lobby Rules (1990) prescrivono che affinché la comunicazione con la base assuma natura di attività lobbistica, deve riferirsi a una specifica legge, riflettere una presa di posizione nel merito della stessa e contenere un «invito all'azione», prospettando specifiche azioni volte ad influenzare la legislazione. L'elaborazione di questa normativa, durata più anni, costituisce in sé un caso riuscito ed importante di lobbying da parte del c.d. Independent Sector, formato da organizzazioni non a scopo di lucro la cui attività richiede rapporti continui coi membri, e che portavano quindi un interesse specialissimo alla materia ${ }^{6}$. Non solo questa «comunità» fece forti pressioni sull'Internal Revenue Service per il tramite di una coalizione denominata Advocacy Forum, ma in un certo senso deve la sua stessa esistenza ai legami e interessi emersi nel corso di questa «lotta». Per dirla con una formula del foglio che serviva da portavoce della coalizione, «c'è voluto l'Internal Revenue Service per farci capire quanto avevamo in comune - e quanto avevamo da perdere» («Advocate's Advocate», ottobre 1988, p. 3).

Ogni associazione di una certa importanza ha una Divisione degli Affari Pubblici (o nome equivalente) che si occupa dei rapporti con la stampa. La stampa - soprattutto i tre-quattro giornali a circolazione nazionale e le più importanti reti TV, ma anche la stampa specialistica (v. oltre) - opera come una sorta di lente d'ingrandimento relativamente ai problemi che preoccupano il gruppo e le posizioni che su di essi il gruppo assume. Il principale scopo non è quello di avere la stampa «dalla propria parte», anche se questo ovviamente non guasta. Forse ancora più importante è la capacità di affermare e mantenere una sorta di monopolio sul discorso politico che tocca i fini dell'as-

${ }^{6}$ I contributi a questi gruppi essendo tax deductible, essi non possono spendere in attività di lobbying più di una frazione delle loro risorse. 
sociazione. Così, ogniqualvolta vengono in discussione temi relativi ai finanziamenti elettorali e la loro regolamentazione, è probabile che sia riportato il punto di vista di Common Cause, un' «autorità» in materia - e ciò a sua volta costituisce una verifica di tale autorevolezza. Lo stesso dicasi per AAU, che «fa opinione» quando vengono in rilievo problemi connessi alla graduate education e alla ricerca.

Per riassumere. L'obiettivo complessivo di un gruppo nei suoi rapporti con il Governo è di assicurarsi che nessuna importante decisione legislativa o amministrativa che incide sugli scopi associativi sia presa senza che il gruppo venga consultato e, cosa più importante, effettivamente sentito. Le risorse discusse nei precedenti paragrafi e il loro uso efficace mirano a stabilire e mantenere nel tempo siffatti «rapporti speciali» con le varie autorità di Governo. Questo è raramente fatto dall'associazione singolarmente: di norma è il risultato di sforzi congiunti da parte del sottosistema lobbistico (o comunità d'interessi) e di altre coalizioni a cui il gruppo può decidere di accedere. Entro tali arene, la regola è la divisione del lavoro: strutturalmente, e cioè sul lungo periodo, ogni associazione cerca di appropriarsi di un pacchetto specifico di issues e priorità che la distinguono da tutte le altre; funzionalmente, per ogni problema o campagna da affrontare di concerto, la posizione di leadership va generalmente a quella con più autorità in rapporto alla materia e più risorse in gioco.

Tratterò brevemente della gratitudine e della fiducia come ingredienti del rapporto. Un elaborato rituale lega il rappresentante alle sue controparti politiche: ogni favore o segno di disponibilità deve essere seguito da note personali di ringraziamento; qualunque iniziativa utile da parte di un Congressman deve trovare eco appropriata nella stampa dell'associazione, con inviti a rivolgersi ai membri in occasione delle riunioni annuali dell'organizzazione, e in altri modi. Due fattori spiegano, credo, questo alto grado di personalizzazione. Uno attiene alla già ricordata natura informale di questo tipo di rappresentanza, che non può essere codificata in termini formali. L'altra ragione è che ciò che è di valore al lobbista - gli schieramenti che si stanno delineando intorno ad un provvedimento, gli emendamenti probabili a una legge a cui bisogna rispondere, e in genere la tempestività delle informazioni - non può essere di norma ottenuto che attraverso contatti altamente personalizzati.

Fiducia, integrità e competenza sono strettamente legate fra 
di loro. L'integrità professionale aiuta a creare quel clima di fiducia che rende possibile e fruttuosa l'interazione. Sebbene siano in genere disponibili ad ascoltare le varie parti, certo con diversi gradi di ricettività a seconda del loro peso politico, i Congressmen, con poche eccezioni, non si possono «comprare»; occorre piuttosto convincerli con risorse politiche. Alcuni dei membri del Congresso con cui ho parlato sembrano fare una distinzione fra «advocacy», l'argomentare esplicitamente di parte a sostegno dei propri obiettivi, e «rappresentanza», propria del lobbista che fornisce informazione obiettiva senza nascondere a nome di chi parla. Per quanto possibile, la persuasione dev'essere esercitata attraverso una discussione sul merito dei problemi ${ }^{7}$, senza sottacere informazioni che potrebbero negativamente influire sulle proprie posizioni. La strategia migliore sembra essere quella di presentare tutti i fatti, compreso il punto di vista degli avversari, fornendo al contempo appropriate contro-argomentazioni.

La fiducia è anche legata ai tempi in base ai quali il lobbista si muove. La regola d'oro, qui come nella vita, è che il momento migliore per fare appello ai propri amici è quando se ne ha meno bisogno. Tenere i canali aperti è essenziale se si vuole preservare un patrimonio su cui poter contare tempestivamente in caso di necessità - e non va dimenticato che parte importante del «capitale» di un lobbista sta nei contatti e rapporti operativi utili che è riuscito a costruire nel tempo. Tutto ciò per reiterare l'osservazione fatta altrove (Graziano 1991), secondo la quale lobbying non è soltanto pressione, ma un processo complesso a più fasi - dallo studio dei dossier alla concertazione di una linea comune all'associazione via via fino alla pressione, che è l'ultimo stadio - processo che di norma richiede una continua presenza organizzata a Washington.

Un ultimo punto in tema di personalizzazione. Sia il lobbying che lo scambio sociale sono rapporti strumentali (v. sopra), ma con un'importante differenza avuto riguardo al grado di libertà nella scelta dei partner. Mi si consenta di richiamare la definizione di scambio in Blau $(1964,6)$ : «Come qui concepito, scambio sociale è limitato ad azioni che dipendono da reazioni remunerative da parte di altri, e che cessano quando queste reazioni non si materializzano». Visto in questa luce, il lob-

7 Mezzi «finanziari» di pressione come i PACs ed altre forme di contributi elettorali possono essere ovviamente molto efficaci, ma esulano dall'ambito di questo saggio. 
bying è una sorta di scambio rigido: il rappresentante deve trattare con pubblici ufficiali che deve accettare più che scegliere, siano essi favorevoli o ostili. Per esemplificare ancora una volta con il caso di AAU: per il presidente e lo staff dell'associazione, il capo e lo staff della National Science Foundation costituiscono partner indispensabili, un «dato», NSF essendo la più importante fonte di fondi per la ricerca accademica di base negli USA; così come, reciprocamente, il personale NSF non ha altra scelta che trattare con la comunità scientifica organizzata e i suoi leader, se vuole mandare ad effetto le proprie politiche.

Il mutuo sostegno fra questi attori è un aspetto importante di ciò che la letteratura chiama «triangoli di ferro» o policy communities - partnerships speciali altamente istituzionalizzate e durevoli nel tempo fra funzionari delle varie Agenzie, dirigenti delle associazioni «clienti» e i membri chiave delle Commissioni del Congresso competenti in materia. In tali reti non è sempre facile separare i vantaggi strumentali da quelli «intrinseci», per usare il linguaggio di Blau. Come ho notato altrove (Graziano 1993, 114), «un linguaggio comune, uno stile di vita simile e spesso amicizia personale, e una comune expertise in una stessa area di politiche pubbliche possono dar vita a una grande affinità fra pubblici funzionari e rappresentanti dei gruppi. Cosa più importante, un comune impegno per l'avanzamento di un servizio o di un'industria e convergenza d'interessi quanto al rafforzamento... della policy community [possono] mettere capo a rapporti spesso al limite della complicità».

Il lato strumentale della partnership può essere brevemente tratteggiato. Da un lato, nel contesto altamente competitivo della politica americana non è nell'interesse del gruppo avere una debole controparte nell'Amministrazione. Un responsabile debole è quasi altrettanto deleterio che un funzionario ostile. Inoltre, è nell'interesse dell'associazione sostenere il bilancio dell'Agenzia, normalmente a livelli più elevati di quelli fissati nel c.d. bilancio del Presidente, che apre a gennaio di ogni anno l'esame del bilancio da parte del Congresso. Ciò vien fatto istituendo coalizioni ad boc in appoggio a specifiche Agenzie di particolare interesse al gruppo ${ }^{8}$, testimoniando davanti ai perti-

8 AAU ha contribuito a fondare e partecipa attivamente a due coalizioni di questo tipo: la Coalition for National Science Funding, che riunisce società scientifiche e professionali, associazioni di università e imprese a sostegno del bilancio annuale della National Science Foundation; l'Ad Hoc Group for Medical Research Funding che svolge analoga azione a favore dei National Institutes of Health e dell'Alcohol, Drug Abuse and Mental Health Administration, agenzia da cui dipende gran parte dei fondi per la ricerca biomedica. 
nenti Appropriations Committees da cui dipende la determinazione ultima dei livelli di spesa, e in altri modi ancora.

L'Agenzia federale risponde tenendo nella massima considerazione e - nella misura del possibile - corrispondendo agli interessi della comunità che la sostiene, specialmente i suoi segmenti più forti. Nel caso della NSF, ciò comporta, fra l'altro, difendere il metodo meritocratico della peer review nella scelta dei progetti da finanziare e nella distribuzione dei fondi di ricerca, metodo che sistematicamente favorisce i centri di ricerca più prestigiosi con le loro superiori risorse, contro ricorrenti richieste d'introdurre criteri più «democratici» (soprattutto quello regionale-territoriale). NSF inoltre spesso sceglie come membri dei comitati consultivi di cui si avvale personale scientifico vicino alle associazioni o appartenente a università che ne sono membri. Non sono, infine, rari i casi di incontri informali nei quali alti esponenti delle Agenzie riferiscono su misure allo studio dell'Amministrazione, questioni di strategia e tattica del Governo e altro, incontri che costituiscono un preziosissimo input e canale per la comunità universitaria e i rappresentanti delle associazioni che vi partecipano.

L'ultimo punto che voglio toccare attiene alla legittimazione. In molti paesi, e segnatamente in Italia, il lobbying e le lobbies evocano un sentimento di vergogna, pratiche corrotte, il lato buio della politica. Non che il sistema sia immune da mende e abusi anche gravi; ma da quanto è dato osservare, opera in America secondo forme e modalità tali da consentire, come si è fatto in questo saggio, di delineare una fisiologia che non ha nulla dei tratti della corruzione e del favoreggiamento sistematico. In ogni caso, anche negli USA lobbying è termine non privo di ambiguità, spesso sostituito con sinonimi più appetibili «educazione politica» (uno dei primi PACs fu creato nel 1943 dal Congress of Industrial Organizations con il nome di Committee on Political Education), advocacy, relazioni federali. Storicamente, tale ambiguità trova riscontro nel laborioso processo attraverso il quale il principio della rappresentanza dei gruppi è stato incorporato nella vita politica americana e nella cultura dominante, processo iniziato a partire dal 1900, culminato durante il New Deal e a tutt'oggi tutt'altro che concluso.

E tuttavia nessun altro paese si è spinto così lontano come gli USA nel riconoscere il lobbismo come istituzione e professione. La sua regolamentazione attraverso la legge (Regulation of Lobbying Act, 1946), basata sul principio della pubblicità 
dei committenti e dei mezzi forniti ai lobbisti, per quanto inadeguata è un primo indicatore di istituzionalizzazione. Lo stesso numero di persone coinvolto è un altro importante fattore: decine di migliaia di persone se si tiene conto dei vari ruoli professionali sussunti sotto il termine lobbying (Graziano 1991) e le loro controparti nel Congresso e nella burocrazia. Questo personale è ormai una componente fondamentale della classe politica, senza la quale la politica americana nella sua presente configurazione sarebbe inimmaginabile. Inoltre, i lobbisti hanno una propria associazione professionale, la American League of Lobbyists, e una deontologia professionale sta emergendo e radicandosi in settori importanti della professione.

Anche qui lo schema di Blau può essere di ausilio. L'idea di Blau è di considerare la legittimazione come il prodotto di cambiamenti nell'ampiezza, complessità e contenuto dello scambio sociale. La distinzione di fondo è al riguardo quella fra scambio diretto di benefici personali, come nello scambio fra patrono e cliente, e scambio indiretto mediato da norme e istituzioni come il moderno sindacato. Man mano che si sviluppano moderne macrostrutture come partiti, imprese, associazioni e altre, anche i valori e le aspettative cambiano: l'equità dello scambio non è più valutata sulla base di vantaggi immediati e personali, come avviene nello scambio diretto, ma piuttosto alla luce di norme di equità la cui formazione è resa possibile dall'emergere di valori e finalità comuni sottesi a tali istituzioni. La legittimazione è dunque un processo collettivo legato alla socializzazione dello scambio, e potrebbe difficilmente emergere in un mondo di transazioni frammentate, interpersonali e diadiche.

Con riferimento al nostro tema, alcune delle condizioni che possono favorire tale processo sono già state menzionate: l'esistenza di una legge che prescrive la registrazione dei lobbisti che operano presso il Congresso e rendiconti periodici con indicazione dei mezzi finanziari coinvolti; audizioni pubbliche presso il Congresso; standard etici legali e statuiti dal Congresso a carico dei propri membri che configurano tutta una serie di casi di «influenza indebita» - tutte queste sono importanti precondizioni di legittimazione. A questi fattori andrebbe aggiunta una cultura politica che, diversamente da quelle europee, non ha mai fatto una distinzione troppo netta fra stato e società civile, rendendo così possibile e favorendo input dalla società e i suoi diversi gruppi organizzati.

Qui posso solo soffermarmi sul contributo delle associazioni 
a questo processo. Essenziale è il ruolo di quelli che ho chiamato sottosistemi lobbistici e policy communities, che socializzano il gioco e costituiscono arene collettive per la sua regolazione sociale. Un aspetto distintivo della comunità lobbistica di Washington, che non può non colpire l'osservatore, è la notevole intensità e frequenza dell'interazione. Esemplifico ancora con il caso della bigher education community, ma le cose non sembrano molto diverse in altri settori. I presidenti delle sei principali associazioni - i Sei Grandi dell'istruzione universitaria (Bloland 1985) - cinque delle quali con sede nello stesso edificio che ospita molti dei gruppi che ruotano intorno a università e ricerca a Washington, si incontrano regolarmente ogni quindici giorni; i direttori dei rapporti federali (i capo-lobbisti di ciascuna associazione) di questi e altri gruppi della «comunità» si riuniscono mensilmente; coalizioni di vario tipo si riuniscono pure regolarmente, come fanno altri funzionari specializzati delle singole associazioni che si ritrovano periodicamente con i loro omologhi delle altre organizzazioni. Degli incontri fra lo staff di gruppi e funzionari di agenzie federali, anch'essi di routine, ho già detto. Mentre la funzione primaria di questi incontri è di condividere e scambiare informazioni, essi forniscono al contempo un foro regolare in cui norme di comportamento collettivo sono socializzate, trasmesse, sperimentate e periodicamente aggiornate.

A ciò va aggiunto il ruolo della stampa specializzata nei singoli settori di politiche pubbliche e altre forme d'interazione, comprese le convenzioni annuali delle diverse associazioni, che sono momenti di socializzazione e scambio intra e inter-organizzativo di grande rilievo. Per restare alla stampa, nel caso della comunità universitaria e della ricerca un certo numero di pubblicazioni, e in particolare l'autorevole e diffuso «Chronicle of Higher Education», contribuiscono a definire profili di comportamento più o meno in linea con norme e standard condivisi. Esempio recente di questa opera di normalizzazione è dato dal caso di una pratica, detta earmarking, consistente nel tentativo spesso coronato da successo di singole università di ottenere fondi dal Congresso attraverso Congressmen amici, evitando il peer review process. Il «Chronicle» ha severamente censurato la pratica, verso cui per ovvie ragioni sono molto critiche anche molte associazioni, svolgendo la funzione di cui sto qui discorrendo. 


\section{Conclusione breve}

Poco è stato detto in questo saggio del background culturale che ha fatto da sfondo all'emergere del lobbying e della politica dei gruppi negli Stati Uniti. La crescita di questo fenomeno riflette, oltre ad importanti cambiamenti nell'economia politica della società americana, profonde forze nella cultura politica di quel paese. Il pluralismo dei gruppi è al contempo in linea e in radicale dissidio rispetto ad alcuni principi basilari del liberalismo, con il gruppo piuttosto che l'individuo e il «popolo» al centro del sistema. In tale trasformazione vi sono certamente pericoli, come ho notato, ma anche potenzialità. Detto brevemente, credo che nel paradigma culturale e normativo entro cui il lobbying è emerso esistano delle potenzialità che normalmente non associamo all'universo pragmatico del lobbismo.

Evocare il pragmatismo è un modo per richiamare il clima ideologico entro il quale la politica dei gruppi si è sviluppata come uno degli aspetti principali del sistema americano di rappresentanza. In effetti, la politica degli interessi è pienamente emersa dopo le elezioni del 1896, secondo molti spartiacque fra la «politica dei partiti» e la «politica pragmatica», e la sconfitta del movimento operaio a fine secolo, che ha deciso la lotta di classe negli Stati Uniti entro i parametri del compromesso capitalista.

Il lobbying è un prodotto di tale «omogeneizzazione» della politica: molte delle sue strategie e l'intero spirito del gioco sarebbero impraticabili in un mondo segnato da profonde divisioni ideologiche. Sinistra e destra hanno scarso significato quando la politica è ridefinita come politica della scienza, tassazione, prevenzione della violenza o finanziamenti elettorali - o meglio, l'insieme di queste issues definiscono opzioni che non permettono di collocare chiaramente gli attori sul continuo destra-sinistra. La stessa logica della rappresentanza degli interessi e le condizioni della sua efficacia, una delle quali è la specializzazione, implicano un radicale divorzio fra politica e ideologia.

D'altro lato, qualunque osservatore non può che essere colpito dalla flessibilità dello strumento. Si può fare attività di lobbying praticamente per qualsiasi interesse o «causa» - sebbene lo strumento non si presti ugualmente bene per tutti i tipi di obiettivi collettivi. Come tale è tecnica che potrebbe rivelarsi utile per il mondo post-ideologico che sembra essere sempre più il nostro, almeno per quella parte dell'azione politica che 
tende a prendere sempre più la forma di aggregazioni ad boc intorno a specifici, ben definiti obiettivi funzionali. Ma, per restare all'interno della cultura che ha visto crescere più rapidamente il fenomeno, va ricordato che lobbying e rappresentanza degli interessi sono parte di una tradizione politica che riconosce tanto l'inevitabilità delle fazioni in una libera vita democratica, che la necessità di limitarne il potere. È la concorrenza, ma anche una «teoria dell'equilibrio civico» che ritroviamo nel background normativo di questo universo.

Resta il problema di sapere se questa forma sia esportabile - e con quali adattamenti - ad altri sistemi. Che la rappresentanza lobbistica sia sempre più frequente a Bruxelles, dove si dice operino già circa 4.000 lobbisti, è un fatto notato da più osservatori. Ma anche nella crisi della politica europea e in particolare italiana, il ridimensionamento del ruolo dei partiti non potrà non aprire spazi crescenti alla politica degli interessi centrata sul gruppo e sull'associazione. Qui non posso che sfiorare l'argomento, che richiederebbe uno studio a parte; lo segnalo solo come uno dei punti di vista importanti da cui valutare la ricostruzione del sistema. Anni prima che l'Italia fosse investita dalla crisi di regime che la scuote, un noto PR italiano (le relazioni pubbliche sono il campo professionale in cui da noi si cerca d'inquadrare il lobbying) aveva notato come corruzione e «parentela» ideologica fossero condizioni che restringevano drasticamente nel nostro paese la possibilità di esplicare una sana attività di pressione. Ed in effetti pressione implica resistenza istituzionale da parte dei decisori che si cerca d'influenzare, una controparte seria che sintetizzi interessi e domande sulla base di criteri e esigenze istituzionali che gli sono propri. Non c'è attività di lobbying necessaria, con la conseguente necessità di provare la fondatezza politica delle proprie argomentazioni con le risorse di cui ho detto, se l'uomo politico può essere «persuaso» con la corruzione o la semplice amicizia.

Per concludere. Lungi dal demonizzarlo, il lobbying va visto, come di fatto vide Madison nelle condizioni della politica del suo tempo, come un aspetto consustanziale alla politica democratica e al repertorio dei suoi strumenti. Ciò è particolarmente vero oggi che gli interessi diventano base sempre più importante della rappresentanza. Un interesse politicamente riconosciuto, e per cui vengono predisposti canali pubblici, è un interesse che avrà tanto meno incentivo - e giustificazione quando tentasse di farlo - ad operare clandestinamente e corrotta- 
mente. $\grave{E}$ anche su questo, oltre che sui nuovi sistemi elettorali, che andranno giudicati gli «ingegneri» del nuovo sistema.

\section{Riferimenti bibliografici}

Baldwin, D.A. (1990), Political Exchange and Cooperation, in B. Marin (1990).

Blau, P.M. (1964), Exchange and Power in Social Life, New York, Wiley.

Bloland, H.G. (1985), Associations in Action. The Washington, D.C., Higher Education Community, Washington, D.C., Association for the Study of Higher Education.

CRS Report (1991), Legal and Congressional Ethics Standards of Relevance to those Who Lobby Congress, Washington, D.C., Congressional Research Service, The Library of Congress.

Friedberg, E. (1990), Generalized Political Exchange, Organizational Analysis, and Public Policy, in B. Marin (1990).

Graziano, L. (1991), Partiti e lobbies: riflessioni sul caso americano, in M. Vaudagna (a cura di), Il partito politico americano e l'Europa, Milano, Feltrinelli.

Graziano, L. (1993), Pluralism in Comparative Perspective, «Politiikka» (Helsinki), vol. II, pp. 108-115.

Marin, B. (a cura di) (1990), Generalized Political Exchange. Antagonistic Cooperation and Integrated Policy Circuits, Francoforte sul Meno, Campus Verlag e Boulder, Westview Press.

Olson, M. (1968), The Logic of Collective Action. Public Goods and the Theory of Groups, New York, Schocken Books; trad. it. La logica dell'azione collettiva, Milano, Feltrinelli, 1983.

O'Neill, Tip (1987), Man of the House. The Life and Political Memoirs of Speaker Tip O'Neill (con W. Novak), New York, Random House.

Pizzorno, A. (1979), Political Exchange and Collective Identity in Industrial Conflict, in C.J. Crouch e A. Pizzorno (a cura di), The Resurgence of Class Conflict in Western Europe since 1968, vol. II, Londra, Macmillan.

Rapporto Bok (1976), Draft Report on the Future of $A A U$, Association of American Universities, ciclostilato.

Salisbury, R.H. (1990), Interest Advocacy and Interest Representation, relazione presentata al Convegno su «Pluralismo e democrazia», Cortona, 29-31 maggio.

Sorauf, F. (1993), Inside Campaign Finance. Myths and realities, New Haven, Yale University Press.

«Washington Representatives» (1992), Washington, D.C.

Zorack, J.L. (1990), The Lobbying Handbook, Washington, D.C., The Professional Lobbying and Consulting Center. 